\title{
Seletividade de Atrazine e Nicosulfuron a Podisus nigrispinus (Heteroptera: Pentatomidae) ${ }^{1}$
}

\author{
Selectivity of Atrazin and Nicosulfurom to Podisus nigrispinus (Heteroptera: Pentatomidae)
}

\author{
MENEZES, C.W.G. ${ }^{2}$, SANTOS, J.B. ${ }^{3}$, ASSIS JÚNIOR, S.L. ${ }^{4}$, FONSECA, A.J. ${ }^{2}$, FRANÇA, A.C. ${ }^{3}$, \\ SOARES, M.A. ${ }^{3}$ e FERNANDES, A.F. ${ }^{5}$
}

\begin{abstract}
RESUMO - O milho tem sido cultivado em duas safras anuais no Brasil e consumido por humanos e animais. O uso de herbicidas no controle de plantas daninhas nessa cultura pode comprometer o ambiente em função dos efeitos sobre organismos não alvos. O objetivo deste trabalho foi avaliar a seletividade de atrazine e nicosulfuron sobre o predador Podisus nigrispinus. Os tratamentos constaram da aplicação de atrazine, nicosulfuron e da mistura destes, em doses equivalentes à comercial, mais um tratamento controle à base de água. A solução com os herbicidas foi aspergida sobre ovos de dois dias de idade de $P$. nigrispinus e em cada um dos cinco estádios ninfais e fase adulta do inseto. A viabilidade dos ovos de $P$. nigrispinus diminuiu sob ação dos herbicidas, sem eles diferirem quanto ao tempo de eclosão. A sobrevivência de ninfas foi baixa sob ação dos herbicidas, sendo mais afetada negativamente sob ação da mistura destes. Para a aplicação em cada estádio, observou-se baixa sobrevivência de ninfas do primeiro ao terceiro estádio sob ação do herbicida atrazine, isolado ou em mistura, e até o segundo estádio para o nicosulfuron. Também ficou evidente em todos os demais estádios do inseto a menor seletividade à mistura dos herbicidas, em comparação ao efeito isolado. Conclui-se que ovos de $P$. nigrispinus são sensiveis aos herbicidas testados e que a aplicação na cultura do milho desses produtos poderá diminuir o controle biológico de pragas promovido por esse inseto.
\end{abstract}

Palavras-chave: Asopinae, controle biológico, pós-emergentes, toxicidade

\begin{abstract}
Corn is traditionally grown in two annual harvests in Brazil and consumed by humans and animals. The use of herbicides to control weeds in this crop may compromise the environment, affecting non-target organisms. The aim of this study was to evaluate the selectivity of atrazine and nicosulfuron on the predator Podisus nigrispinus Dallas, 1851 (Heteroptera: Pentatomidae). The treatments consisted of application of a combination of atrazine and nicosulfuron in doses equivalent to the commercial dose, plus a water-based control. The solution with herbicides was sprayed on two-day-old eggs of $\boldsymbol{P}$. nigrispinus and at each of the five nymphal stages and adult phase of the insects. The viability of eggs of $\boldsymbol{P}$. nigrispinus decreased under the action of the herbicides, without differences regarding time of hatching. The survival of nymphs was low under the effect of the herbicides, being more adversely affected by the action of the herbicide mixtures. Forthe application at each stage, we observed a low survival rate of nymphs from the first to the third instar under the action of the herbicide atrazine, alone, or in combination, and even at the second stage for nicosulfuron. Lower selectivity to herbicide mixture also became evident at all the insect stages, compared to the isolated effect. It was concluded that eggs of $\boldsymbol{P}$. nigrispinus are sensitive to herbicides and that application of such products on corn may decrease the biological pest control provided by this insect.
\end{abstract}

Keywords: Asopinae, biological control, post emergent, toxicity.

1 Recebido para publicação em 20.4.2011 e aprovado em 26.02.2012.

2 Mestrandos do Programa de Pós-Graduação em Produção Vegetal, Universidade Federal dos Vales do Jequitinhonha e Mucuri PPGPV/UFVJM, <claubertmenezes@yahoo.com.br>, <arley.agro@yahoo.com.br>; ${ }^{3}$ Docentes, PPGPV/UFVJM, $<$ barbosa@pq.cnpq.br>, <cabralfranca@yahoo.com.br>, <marcusasoares@yahoo.com.br>; ${ }^{4}$ Docente do Programa de Pós-Graduação em Ciência Florestal - PPGCF/UFVJM, <assisjr@ufvjm.edu.br>; ${ }^{5}$ Mestranda do Programa de Pós Graduação em Fitopatologia, Universidade Federal de Viçosa - UFV, <alessandradtna@yahoo.com.br>.

Planta Daninha, Viçosa-MG, v. 30, n. 2, p. 327-334, 2012 


\section{INTRODUÇÃO}

A cultura do milho é uma das mais importantes no Brasil, ocupando 13 milhões de hectares, com produção de 52 milhões de toneladas por ano (Conab, 2010). O manejo de insetos-praga como a lagarta-do-cartucho Spodoptera frugiperda e a lagarta-da-espiga Helicoverpa zea, as quais causam danos diretos e indiretos e perdas na produção, e o controle de plantas daninhas assumem grande parcela entre os custos de produção (Constantin et al., 2007; Stefanello Júnior et al., 2008).

As plantas daninhas são responsáveis pelas perdas na qualidade e rendimento de grãos e, no caso do milho, podem inviabilizar a colheita (Constantin et al., 2007). Nesse sentido, o uso de herbicidas é prática comum, em razão do menor custo e da maior eficiência de controle (Rizzardi et al., 2004). Contudo, a diversidade dessas plantas daninhas em campo pode ser um importante fator para o estabelecimento e a dinâmica de inimigos naturais de pragas, onde esses insetos usufruem dessas plantas para refúgio e sobrevivência (Evangelista Jr. et al., 2003). Os herbicidas atrazine e nicosulfuron são produtos usados atualmente em razão do baixo custo e seletividade à cultura (Rizzardi et al., 2004), sendo recomendados, respectivamente, para controle de plantas daninhas de folhas largas e gramineas (Brasil, 2010).

O herbicida atrazine é o mais conhecido dentro da classe das triazinas. Seu uso para fins agrícolas é autorizado no Brasil, podendo ser aplicado em pré e pós-emergência em plantas daninhas nas culturas de abacaxi, cana-de-açúcar, milho, pínus, seringueira, sisal e sorgo (Brasil, 2010). Seu mecanismo de ação se dá pela inibição do fotossistema II, causando uma série de danos irreversiveis às células vegetais. É um herbicida não sistêmico, exercendo controle eficiente de dicotiledôneas e regular de monocotiledôneas. Apresenta mecanismo de seletividade para certas culturas, devido à degradação diferencial, em que nas raízes ou em outras partes ocorre a metabolização e transformação rápida em produtos não tóxicos para as plantas (Carvalho et al., 2010). Apresenta meia-vida no solo entre 15 e 100 dias, porém, devido ao seu largo uso em todo o mundo e à baixa capacidade adsortiva em solos, é um dos herbicidas mais lixiviados e danosos ao meio ambiente (Ralebitso et al., 2002).

O nicosulfuron é um herbicida sistêmico, pós-emergente, do grupo químico das sulfonilureias, que se destaca pela ampla utilização na cultura do milho, no controle de gramíneas e algumas dicotiledôneas. Herbicidas desse grupo atuam na inibição da acetolactato sintase (ALS) em plantas e microrganismos, prejudicando a biossintese de aminoácidos de cadeia ramificada: valina, leucina e isoleucina (Anderson et al., 1998). Esse herbicida é normalmente misturado com outros agroquímicos, apresentando efeitos negativos ao meio ambiente, como relatado em diversos trabalhos (Silva et al., 2005; Oliveira et al., 2009).

Percevejos do gênero Podisus spp. constituem-se em importantes agentes de controle biológico no Brasil (Vivian et al., 2002). O predador Podisus nigrispinus é importante no Manejo Integrado de Pragas no milho, pelo seu desempenho como agente de controle biológico, alimentando-se principalmente de lagartas desfolhadoras (Mohaghegh et al., 2001; Matos Neto et al., 2002). Trabalhos têm evidenciado $P$. nigrispinus se alimentando de lepidópteras-praga, como $S$. frugiperda, resultando em ganhos para diferentes variáveis de desenvolvimento biológico e reprodução, demonstrando seu potencial como importante controle biológico dessa lagarta (Oliveira et al., 2004; Zanuncio et al., 2008). Esse inseto apresenta atividade de predação e acasalamento em período diurno e noturno, podendo estar exposto ao contato direto e residual desses herbicidas na cultura durante a pulverização convencional (Stefanello Júnior et al., 2008). Ademais, a mistura desses herbicidas poderá vir a ocasionar efeito sinergético de toxicidade a $P$. nigrispinus,

A intoxicação de insetos por pesticidas foi relatada por Giolo et al. (2005) e Tillman (2006), justificando a necessidade de estudos de seletividade de herbicidas a inimigos naturais. Além disso, alguns surfatantes presentes em formulações comerciais podem reduzir a tensão superficial no tegumento dos organismos, facilitando sua penetração à ação tóxica desse ingrediente ou do herbicida, ou ainda a ação conjunta da mistura comercial 
(Malkones, 2000). O objetivo deste trabalho foi avaliar a seletividade dos herbicidas atrazine e nicosulfuron, aplicados em ovos e diferentes estádios de desenvolvimento de $P$. nigrispinus, na taxa de eclosão e mudança de estádio ninfal até a fase adulta.

\section{MATERIAL E MÉTODOS}

O trabalho foi realizado em condições controladas $\left(25 \pm 2{ }^{\circ} \mathrm{C}, 70 \pm 10 \%\right.$ de umidade relativa e fotoperíodo de 12 horas).

Foram realizados dois ensaios para aplicação dos herbicidas, sendo o primeiro utilizando posturas com dois dias de idade de $P$. nigrispinus, que foram coletadas de insetos adultos alimentados com pupas de Tenebrio molitor e água destilada. No segundo ensaio, ninfas do primeiro ao quinto estádio de desenvolvimento e insetos adultos de $P$. nigrispinus foram retirados de criação massal em laboratório e submetidos à pulverização dos herbicidas.

Para o primeiro ensaio, os tratamentos, em número de quatro e dispostos em delineamento inteiramente casualizado, foram compostos pelos herbicidas atrazine (Primóleo $\mathbb{R}$ ) e nicosulfuron (Sanson $\mathbb{R}$ ), aplicados isolados ou em mistura, mais um tratamento controle à base de água destilada, diretamente sobre os ovos do inseto. Na aplicação utilizou-se uma seringa de $1 \mathrm{~mL}$, com agulha adaptada para pulverização, aspergindo-se $0,15 \mathrm{~mL}$, calculado de acordo com o diâmetro da placa de Petri, onde foram dispostos os ovos, de calda equivalente a $200 \mathrm{~L} \mathrm{ha}^{-1}$. Utilizou-se a concentração de 0,3 e $15 \mathrm{~g} \mathrm{~L}^{-1}$, respectivamente, para atrazine e nicosulfuron, correspondentes à recomendação de 1,5 e $6 \mathrm{~L} \mathrm{ha}^{-1}$ dos produtos comerciais (Brasil, 2010).

Em cada placa de Petri foram dispostos dez ovos de $P$. nigrispinus, constituindo a unidade amostral com cinco repetições. A cada dia foi realizada a contagem de ovos eclodidos, e as ninfas oriundas dos ovos tratados com os herbicidas foram acompanhadas até a fase adulta. Ao atingirem o segundo estádio, as ninfas foram transferidas para potes plásticos de $500 \mathrm{~mL}$ e alimentadas com dieta à base de pupas de T. molitor e água destilada, onde se observou a mudança de estádio desde a fase de eclosão até a adulta. Ao final do período ninfal, encerrou-se a avaliação e foram obtidos os resultados de sobrevivência em cada estádio.

Quanto ao segundo ensaio, empregou-se o esquema fatorial, sendo cada estádio do inseto (primeiro ao quinto e adulto) avaliado sob o efeito dos herbicidas atrazine e nicosulfuron em concentração e esquema de aplicação conforme descrito para o primeiro ensaio. Além da aplicação isolada de cada herbicida, realizou-se a verificação do efeito da mistura e do tratamento controle à base de água destilada, totalizando 24 tratamentos dispostos ao acaso. Contudo, os herbicidas foram aspergidos, conforme metodologia exposta no ensaio 1 , em potes de plástico de $500 \mathrm{~mL}$, contendo, cada um, dez ninfas de $P$. nigrispinus para cada estádio, constituindo a unidade amostral, com cinco repetições. Foi aspergido $0,15 \mathrm{~mL}$, calculado de acordo com a área dos potes, de calda equivalente a $200 \mathrm{~L} \mathrm{ha}^{-1}$. Utilizou-se a concentração de 15 e $0,3 \mathrm{~g} \mathrm{~L}^{-1}$, respectivamente, para atrazine e nicosulfuron, correspondentes à recomendação de $6 \mathrm{e}$ $1,5 \mathrm{~L} \mathrm{ha}^{-1}$ dos produtos comerciais (Brasil, 2010). Para a fase adulta, foram coletados cinco insetos fêmeas e cinco machos de três dias de idade, para cada unidade amostral. Os insetos foram alimentados com pupas de T. molitor e água destilada, fornecidos diariamente até o fim do experimento. A cada dia foi realizada a contagem dos insetos mortos e avaliada a sobrevivência até o período de 96 horas após aplicação dos produtos.

Os dados foram submetidos aos testes das pressuposições do modelo matemático (normalidade e homogeneidade das variâncias) e, em seguida, à análise de variância, sendo as médias, quando significativas, comparadas pelo teste de Duncan a 5\% de probabilidade.

\section{RESULTADOS E DISCUSSÃO}

Para o ensaio com ovos de P. nigrispinus, não houve diferença estatística no número de dias entre a aplicação dos herbicidas e a eclosão de ovos. No entanto, os herbicidas promoveram diminuição acentuada na porcentagem de ovos eclodidos (Tabela 1).

Enquanto no controle a viabilidade de ovos eclodidos foi de $76 \%$, nas parcelas com a mistura dos herbicidas esse valor caiu para $20 \%$. 
Com a aplicação isolada dos produtos, o valor da eclosão foi, em média, de $28 \%$ (Tabela 1 ).

Tabela 1 - Tempo decorrido entre as aplicações dos herbicidas atrazine e nicosulfuron para a eclosão e percentual de indivíduos eclodidos de Podisus nigrispinus (Heteroptera: Pentatomidae). UFVJM, 2010/11

\begin{tabular}{|l|c|c|}
\hline \multicolumn{1}{|c|}{ Tratamento } & Tempo (dias) & Eclosão (\%) \\
\hline Atrazine & $4,60 \mathrm{a}$ & $26,00 \mathrm{~b}$ \\
\hline Nicosulfuron & $5,40 \mathrm{a}$ & $30,00 \mathrm{~b}$ \\
\hline Mistura $^{1 /}$ & $6,20 \mathrm{a}$ & $20,00 \mathrm{c}$ \\
\hline Controle $^{2 /}$ & $5,00 \mathrm{a}$ & $76,00 \mathrm{a}$ \\
\hline
\end{tabular}

${ }^{1 /}$ mistura de atrazine e nicosulfuron; ${ }^{2 /}$ controle (água). Médias seguidas de mesma letra, na coluna, não diferem pelo teste de Duncan a $5 \%$ de probabilidade.

Os menores valores observados na eclosão podem ser devido ao efeito direto dos herbicidas, dos aditivos presentes na formulação comercial ou de ambos sobre os ovos de $P$. nigrispinus. Os surfatantes - nas formulações comerciais de defensivos, inclusive herbicidas - reduzem a tensão superficial promovida por ceras e outros componentes em cutículas de plantas e facilitam a penetração dos produtos em dermes de organismos não alvo, como insetos (Hassan et al., 2000).
No que se refere ao desenvolvimento das ninfas de $P$. nigrispinus oriundas dos ovos eclodidos, verificou-se efeito dos herbicidas entre os estádios I e IV, a partir do qual não houve mais mortes provocadas pelos herbicidas até a fase adulta (Figura 1 e Tabela 2).

Existem poucos estudos investigando a atividade de pesticidas sobre ovos de heterópteros. Porém, pesquisa feita com um regulador de crescimento de insetos, novaluron, que atua por inibição da sintese de quitina durante a muda de estádio ninfal (ecdise), aplicado sobre ovos de Podisus maculiventris (Say) (Heteroptera: Pentatomidae), mostrou ser ele tóxico ao longo do tempo, diminuindo de forma considerável emergentes dos primeiros estádios desse inseto (Cutler et al., 2006).

Comparação realizada entre os diferentes estádios evidenciou queda natural na sobrevivência após o segundo estádio, ou seja, mesmo na ausência dos herbicidas, houve pequena taxa de mortalidade nessa fase (Figura 1).

Entre os herbicidas, é mais fácil entender o efeito tóxico quando se estima a sobrevivência relativa em cada estádio, proposta na Tabela 2 .

A comparação dos tratamentos em cada estádio evidenciou igualdade de efeitos entre

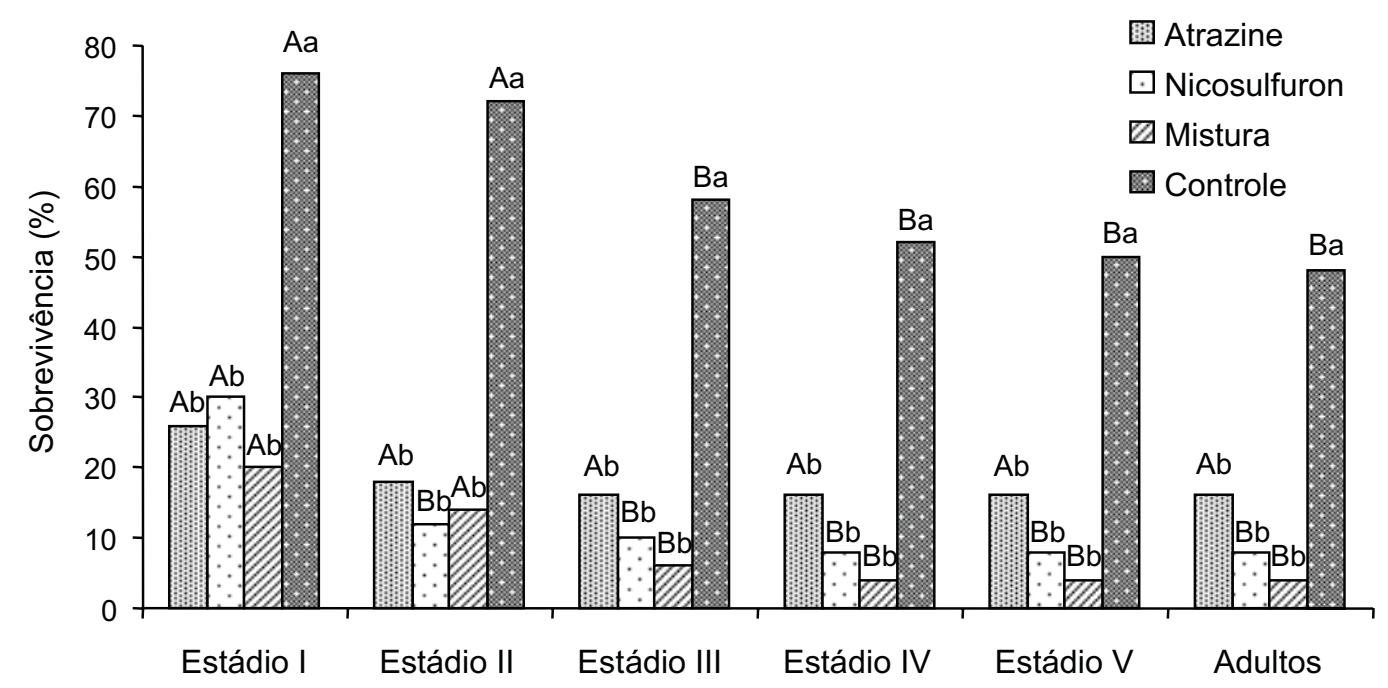

Médias de indivíduos sobreviventes entre os estádios por tratamento, seguidas de mesma letra maiúscula sobre as barras, não diferem pelo teste de Duncan a 5\% de probabilidade; médias de indivíduos sobreviventes em cada estádio, seguidas de mesma letra minúscula, não diferem pelo teste de Duncan a $5 \%$ de probabilidade.

Figura 1 - Sobrevivência (\%) dos estádios de Podisus nigrispinus após aplicação de atrazine e nicosulfuron - isolados ou em mistura e controle (água). 
o controle e o nicosulfuron para os indivíduos que passaram do primeiro para o segundo estádio (Tabela 2). Apesar disso, esse herbicida não apresentou diferenças dos valores observados para o atrazine isolado ou em mistura.

Entre os estádios, para cada herbicida, a comparação revelou efeito negativo mais prolongado para a mistura dos produtos, afetando negativamente até o quarto estádio ninfal (Tabela 2). Assim, entende-se que, quando aplicados juntos, atrazine e nicosulfuron têm efeito residual maior no organismo dos insetos, comparado ao efeito isolado de cada composto.

Em trabalho realizado sobre o impacto de herbicidas em artrópodes do solo, verificou-se que o atrazine e nicosulfuron apresentaram efeito nocivo ao artrópode Collembola, influenciando na abundância desse inseto no solo e reduzindo sua população (Vilma et al., 2007). Também já foi relatada a redução nas populações de ácaros e formigas do solo em virtude da aplicação da mistura dos herbicidas atrazine e nicosulfuron (Pereira et al., 2004). É provável que os efeitos específicos das formulações comerciais testadas no desenvolvimento de $P$. nigrispinus sejam decorrentes da presença de diferentes substâncias químicas na formulação dos herbicidas, como solventes, surfatantes e agentes molhantes, que podem modificar o efeito do equivalente ácido do herbicida nos organismos (Malkones, 2000).

O efeito da aplicação direta dos herbicidas em cada fase de desenvolvimento de $P$. nigrispinus resultou na menor sobrevivência de ninfas do primeiro ao terceiro estádio decorrido o tempo de 96 horas (Tabela 3). Em trabalhos com inseticidas, verificou-se alta toxicidade a ninfas de $P$. nigrispinus expostas ao neonicotinoide imidaclopride, em manejo de pragas na cultura do algodão (Torres \& Ruberson, 2004). Insetos de segundo e terceiro estádios de $P$. nigrispinus apresentaram alta mortalidade devido à exposição dos inseticidas imidaclopride e ao piretroide (betaciflutrina), o que pode ser atribuído ao contato do predador com os resíduos (Karimzadeh et al., 2004; Sarfraz et al., 2008). Assim, podem-se associar os resultados encontrados à menor tolerância da cutícula dos insetos nessa fase inicial de desenvolvimento.

Tabela 2 - Sobrevivência relativa (\%) de Podisus nigrispinus após mudança de estádio em ambiente com resíduos dos herbicidas atrazine e nicosulfuron

\begin{tabular}{|l|c|c|c|c|c|c|}
\hline \multirow{2}{*}{ Tratamento ${ }^{\mathbf{1}}$} & \multicolumn{5}{c|}{ Estádio } \\
\cline { 2 - 7 } & I & II & III & IV & V & Adulto \\
\hline Atrazine & $26,0 \mathrm{Bc}$ & $75,0 \mathrm{Bb}$ & $93,3 \mathrm{Aa}$ & $100,0 \mathrm{Aa}$ & $100,0 \mathrm{Aa}$ & $100,0 \mathrm{Aa}$ \\
\hline Nicosulfuron $^{*}$ & $30,0 \mathrm{Bc}$ & $83,3 \mathrm{ABb}$ & $80,0 \mathrm{Ab}$ & $90,0 \mathrm{Aa}$ & $100,0 \mathrm{Aa}$ & $100,0 \mathrm{Aa}$ \\
\hline Mistura $^{1 /}$ & $20,0 \mathrm{Cc}$ & $66,7 \mathrm{Bb}$ & $70,0 \mathrm{Ab}$ & $80,0 \mathrm{Ab}$ & $100,0 \mathrm{Aa}$ & $100,0 \mathrm{Aa}$ \\
\hline Controle $^{2 /}$ & $76,0 \mathrm{Aa}$ & $95,5 \mathrm{Aa}$ & $81,1 \mathrm{Aa}$ & $90,9 \mathrm{Aa}$ & $96,0 \mathrm{Aa}$ & $96,0 \mathrm{Aa}$ \\
\hline
\end{tabular}

${ }^{1 /}$ mistura de atrazine e nicosulfuron; ${ }^{2 /}$ controle (água). Médias seguidas de mesma letra, na coluna e linha, não diferem pelo teste de Duncan a $5 \%$ de probabilidade.

Tabela 3 - Sobrevivência (\%) de Podisus nigrispinus do primeiro estádio ninfal à fase adulta, em laboratório, após 96 horas de exposição aos herbicidas atrazine e nicosulfuron e à mistura destes

\begin{tabular}{|c|c|c|c|c|c|c|}
\hline \multirow{2}{*}{ Tratamento $^{\underline{1}}$} & \multicolumn{6}{|c|}{ Estádio } \\
\hline & I & II & III & IV & V & Adulto \\
\hline Atrazine & $8 \mathrm{c}$ & $18 \mathrm{c}$ & $42 \mathrm{~b}$ & $50 \mathrm{~b}$ & $74 \mathrm{~b}$ & $82 \mathrm{~b}$ \\
\hline Nicosulfuron & $48 \mathrm{~b}$ & $66 \mathrm{~b}$ & $50 \mathrm{~b}$ & $90 \mathrm{a}$ & $84 \mathrm{ab}$ & $98 \mathrm{a}$ \\
\hline Mistura $^{1 /}$ & $2 \mathrm{c}$ & $6 c$ & $24 \mathrm{c}$ & $36 \mathrm{~b}$ & $38 \mathrm{c}$ & $40 \mathrm{c}$ \\
\hline Controle $^{2 /}$ & $68 \mathrm{a}$ & $84 \mathrm{a}$ & $76 \mathrm{a}$ & $98 \mathrm{a}$ & $94 \mathrm{a}$ & $98 \mathrm{a}$ \\
\hline
\end{tabular}

${ }^{1 /}$ mistura de atrazine e nicosulfuron; ${ }^{2 /}$ controle (água). Médias seguidas de mesma letra, na coluna, não diferem pelo teste de Duncan a $5 \%$ de probabilidade. 
A espessura e o teor de lipídios da cutícula de insetos podem influenciar na penetração de pesticidas, como observado em inseticidas piretroides, que demonstraram menor toxicidade ao percevejo predador $P$. maculiventris e mais nocivo à lagarta Anticarsia gemmatalis (Baptista et al., 1995). Em Brontocoris tabidus, a rede traqueal, que supre as gônadas de oxigênio, consistiu, provavelmente, na principal via de intoxicação, expondo essas estruturas diretamente aos voláteis do piretroide deltamentrina (Vargas, 2007).

O percevejo-verde (Nezara viridula), praga da soja, e o inimigo natural $P$. maculiventris foram expostos a resíduos do inseticida lambdacyhalothrin; $N$. virudula foi mais suscetivel à intoxicação do que $P$. maculiventris, mesmo ambos sendo da mesma família e aparentemente consistirem de similar cutícula esclerotizada (Vandekerkhove \& Clerck, 2004). Mais pesquisas devem ser feitas para elucidar o mecanismo de ação de pesticidas, sobretudo herbicidas, por contato sobre insetos não alvos.

Apesar de o índice de mortalidade utilizado para avaliar a periculosidade de um produto químico não ser a única nem a melhor forma de condução do trabalho (Stark \& Banks, 2003), pois indivíduos sobreviventes aos tóxicos podem apresentar esterilidade (Carson, 1962), essa característica pode indicar o efeito instantâneo dos produtos sobre a população desses insetos (Teodoro et al., 2005).

A mistura dos herbicidas atrazine e nicosulfuron demonstrou maior efeito tóxico para todos os estádios do predador, promovendo sobrevivência média inferior a 50\%. Alguns trabalhos têm demonstrado efeito inseticida de alguns herbicidas em predadores naturais, reduzindo a população desses insetos (Soares et al., 1995).

A partir do quarto estádio ninfal até a fase adulta de $P$. nigrispinus, a taxa de sobrevivência foi maior, possivelmente devido à maior tolerância aos herbicidas. Ninfas de $P$. nigrispinus no quinto estádio demonstraram menor sensibilidade quando submetidas à aplicação de uma dose comercial de inseticidas (Suinaga et al., 1996). O herbicida nicosulfuron foi mais seletivo aos insetos a partir do segundo estádio, permitindo a sobrevivência média superior a $50 \%$, e na fase adulta, equiparando-se aos efeitos do tratamento controle, após 96 horas (Tabela 3).

Conclui-se que a viabilidade dos ovos de $P$. nigrispinus diminuiu sob ação dos herbicidas, sem eles diferirem quanto ao tempo de eclosão. A taxa de sobrevivência de $P$. nigrispinus foi afetada negativamente pelos herbicidas até no quarto estádio ninfal. Quanto à aplicação em cada estádio, observou-se baixa sobrevivência de ninfas do primeiro ao terceiro estádio sob ação do herbicida atrazine, isolado ou em mistura. Também ficou evidente em todos os demais estádios do inseto a menor seletividade à mistura dos herbicidas, em comparação ao efeito isolado. Insetos do quarto estádio até a fase adulta demonstraram maior tolerância aos herbicidas.

\section{AGRADECIMENTOS}

À Fundação de Amparo à Pesquisa do Estado de Minas Gerais (FAPEMIG), ao Conselho Nacional de Desenvolvimento Científico e Tecnológico (CNPq) e à Coordenação de Aperfeiçoamento de Pessoal de Nivel Superior (CAPES); pelo financiamento e suporte.

\section{LITERATURA CITADA}

ANDERSON, D. D.; NISSEN, S. J.; MARTIN, A. R Mechanism of primisulfuron resistance in a shattercane (Sorghum bicolor) biotype. Weed Sci., v. 46, n. 1, p. 158-162, 1998.

BAPTISTA, G. C.; PARRA, J. R. P.; HADDAD, M. L. Toxicidade comparativa de lambda-cyhalothrin à lagarta-dasoja, Anticarsia gemmatalis Hueb., 1818 (Lepidoptera, Noctuidae) e ao percevejo verde, Nezara viridula (L., 1758) (Hemiptera, Pentatomidae). Sci. Agric., v. 52, n. 1, p. $183-188,1995$.

CARSON, R. Primavera silenciosa. São Paulo: Melhoramento, 1962. p. 305.

CARVALHO, F. T.; MORETTI, T. B.; SOUZA, P. A. Eficácia e seletividade de associações de herbicidas utilizados em pós-emergência na cultura do milho. R. Bras. Herb., v. 9, n. 1, p. $35-41,2010$.

COMPANHIA NACIONAL DE ABASTECIMENTO CONAB. Acompanhamento de safra brasileira: Grãos, Quarto levantamento, janeiro de 2010 / Brasília. Disponível em: <www.conab.gov.br/OlalaCMS/uploads/arquivos/ 11_01_06_08_41_56_boletim_graos_4o_lev_safra_2010_2011..pdf >> Acesso em: $\overline{13}$ jan. 2011. 
CONSTANTIN, J. et al. Interação entre sistemas de manejo e de controle de plantas daninhas em pós-emergência afetando o desenvolvimento e a produtividade do milho. Planta

Daninha, v. 25, n. 3, p. 513-520, 2007

CUTLER, G. C. et al. Toxicity of the insect growth regulator novaluron to the non-target predatory bug Podisus maculiventris (Heteroptera: Pentatomidae). Biol. Control, v. 38, n. 1, p. 196-204, 2006.

EVANGELISTA JR, W. S. et al. Efeito de plantas daninhas e do algodoeiro no desenvolvimento, reprodução e preferência para oviposição de Podisus nigrispinus (Dallas) (Heteroptera Pentatomidae). Neotr. Entomol., v. 32, n. 4, p. 677-684, 2003.

GIOLO, F. P. et al. Seletividade de formulações de glyphosate a Trichogramma pretiosum (Hymenoptera:

Trichogrammatidae). Planta Daninha, v. 23, n. 3, p. $457-462,2005$.

HASSAN, S. A. et al. laboratory method to evaluate the side effects of plant protection products on Trichogramma cacoeciae Marchal (Hym., Trichogrammatidae). In: CANDOLFI, M. P. et al. Guidelines to evaluate sideeffects of plant protection products to non-target arthropods. Reinheim: IOBC/WPRS, 2000. p. 107-119.

KARIMZADEH, J.; BONSALL, M. B.; WRIGHT, D. J. Bottom-up and top-down effects in a tritrophic system: the population dynamics of Plutella xylostella (L.) - Cotesia plutellae (Kurdjumov) on different host plants. Ecol.

Entomol., v. 29, p. 285-293, 2004.

MALKONES, H. P. Comparison of the effects of differently formulated herbicides on soil microbial activities: a review. J. Plant Dis. Protec., v. 8, n. 5, p. 781-789, 2000.

BRASIL. Ministério da Agricultura, Pecuária e Abastecimento. Consulta de Produtos Formulados/2010 Disponível em: $<$ http://agrofit.agricultura.gov.br/agrofit_cons/ principal_agrofit_cons $>$ Acesso em: 13 jan. 2011.

MATOS NETO, F. C. et al. Nynphal development of Podisus nigrispinus (Heteroptera, Pentatomidae) preying on larvae of Anticarsia gemmatalis (Lepdoptera, Noctuidae) Fed with resistant e susceptible soybens. R. Bras. Entomol., v. 46, n. 3 , p. $237-241,2002$.

MOHAGHEGH, J.; DE CLERCQ, P.; TIRRY, L. Functional response of the predators Podisus maculiventris (Say) and Podisus nigrispinus (Dallas) (Heteroptera: Pentatomidae) to the beet armyworm, Spodoptera exigua (Hubner) (Lepidoptera: Noctuidae): effect of temperature. J. Appl. Entomol., v. 125, n. 1, p. 131-134, 2001.
OLIVEIRA, A. T. et al. Efeito da interação do nicosulfuron e Chlorpyrifos sobre o banco de sementes e os atributos microbianos do solo. R. Bras. Ci. Solo, v. 33, n. 3, p. $563-570,2009$.

OLIVEIRA, H. N. et al. Desenvolvimento do predador Podisus nigrispinus alimentado com Spodoptera frugiperda $\mathrm{e}$ Tenebrio molitor. Pesq. Agropec. Bras., v. 39, n. 10, p. 947-951, 2004.

PEREIRA, J. L. et al. Effects of herbicide and insecticide interaction on soil entomofauna under maize crop. J.

Environ. Sci. Health, Part B: Pestic., Food Contam., Agric. Wastes, v. 40, n. 1, p. 43-52, 2004.

RALEBITSO, T. K.; SENIOR, E.; VERSEVELD, H. W. V. Microbial aspects of atrazine degradation in natural environments. Biodegradation, v. 13, n. 1, p. 11-19, 2002.

RIZZARDI, M. A.; KARAM, D.; CRUZ, M. B. Manejo e controle de plantas daninhas em milho e sorgo. In: VARGAS, L.; ROMAN, E. S. (Eds.). Manual de manejo e controle de plantas daninhas. Bento Gonçalves: Embrapa Uva e Vinho, 2004. p. 571-594.

SARFRAZ M.; DOSDALL, L. M.; KEDDIE, B. A. Host plant genotype of the herbivore Plutella xylostella (Lepidoptera: Plutellidae) affects the performance of its parasitoid Diadegma insulare (Hymenoptera: Ichneumonidae). Biol. Control, v. 44, n. 1, p. 42-51, 2008.

SILVA, A. A. et al. Efeitos de mistura de herbicida com inseticida sobre cultura do milho, as plantas daninhas e a lagarta-do cartucho. Planta Daninha, v. 23, n. 3, p. 517-525, 2005.

SOARES, J. J.; BRAZ, B. A.; BUSOLI, A. C. Impacto de herbicidas sobre artrópodos benéficos associados ao algodoeiro. Pesq. Agropec. Bras., v. 30, n. 9, p. 1135-1140, 1995.

STARK, J. D.; BANKS, J. E. Population-level effects of pesticides and other toxicants on arthropods. Ann. Rev. Entomol., v. 48, n. 1, p. 505-519, 2003.

STEFANELLO JÚNIOR, G. J. et al. Seletividade de herbicidas registrados para a cultura do milho a adultos de Trichogramma pretiosum (Hymenoptera: Trichogrammatidae). Planta Daninha, v. 26, n. 2, p. 343-351, 2008.

SUINAGA, F. A. et al. Seletividade fisiológica de inseticidas a Podisus nigrispinus (Dallas, 1851) (Heteroptera: Pentatomidae) predador de lagartas desfolhadoras de eucalipto. R. Árvore, v. 20, n. 3, p. 407-414, 1996. 
TEODORO, A. V. et al. Lethal and sub-lethal selectivity of fenbutatin oxide and sulfur to the predador Iphiseiodes zuluagai (Acari: Phytoseiidae) and its prey, Olygonycgus ilicis (Acari: Tetranychidae), in Brazilian coffee plantations. Exper. Appl. Acarol., v. 36, n. 1, p. 61-70, 2005.

TILLMAN, P. G. Susceptibility of pest Nezara viridula (Heteroptera: pentatomidae) and parasitoid Trichopoda pennipes (Diptera: Tachinidae) to selected inseticides. J. Econ. Entomol., v. 99, n. 3, p. 648-657, 2006.

TORRES, J. B.; RUBERSON, J. R. Toxicity of thiamethoxam and imidacloprid to Podisus nigrispinus (Dallas)

(Heteroptera: Pentatomidae) nymphs associated to aphid and whitefly control in cotton. Neotr. Entomol., v. 33, n. 1, p. $99-106,2004$

VANDEKERKHOVE, B. \& DE CLERCQ, P. Effects of an encapsulated formulation of lambda-cyhalothrin on Nezara viridula and its predator Podisus maculiventris (Heteroptera: Pentatomidae). Florida Entomol., v. 87, n. 1, p. 112-118, 2004.
VARGAS, P. S. R. Modificações do aparelho reprodutor de Brontocoris tabidus (Heteroptera: Pentatomidae) após exposição à deltametrina. 2007. 51 f. Dissertação (Mestrado em Entomologia Agrícola) - Universidade Federal de Viçosa, Viçosa, MG, 2007.

VILMA, S. L.; SANTOS, H. R.; GONÇALVES, M. C. The effect of the glyphosate, 2,4-D, atrazine e nicosulfuron herbicides upon the edaphic Collembola (Arthropoda: Ellipura) in a no tillage system. Neotr. Entomol., v. 36, n. 1, p. $261-267,2007$

VIVIAN, L. M. et al. Tasa de crecimiento poblacional del chinche depredador Podisus nigrispinus (Heteroptera: Pentatomidae) y de la presa Tuta absoluta (Lepidoptera: Gelechiidae) en invernadero. R. Biol. Trop., v. 50, n. 1, p. 145-153, 2002.

ZANUNCIO, J. C. et al. Predation rate of Spodoptera frugiperda (Lepidoptera: Noctuidae) larvae with and without defense by Podisus nigrispinus (Heteroptera: Pentatomidae). Braz. Arch. Biol. Technol., v. 51, n. 1, p. 121-125, 2008. 